\title{
Study on the Dynamic Prediction Method of ECD in Horizontal Well Drilling
}

\author{
Ma Xianming ${ }^{1}$, Guan Zhichuan ${ }^{1,}$,, Luo Ming ${ }^{2}$, Li Wentuo ${ }^{2}$, Pan Rui ${ }^{1}$ \\ ${ }^{1}$ School of Petroleum Engineering, China University of Petroleum, Qingdao, China \\ ${ }^{2}$ Zhanjiang Branch, CNOOC China Limited, Zhanjiang, China
}

Email address:

568238622@qq.com (Ma Xianming), guanzhch $a$ upc.edu.cn (Guan Zhichuan)

${ }^{*}$ Corresponding author

\section{To cite this article:}

Ma Xianming, Guan Zhichuan, Luo Ming, Li Wentuo, Pan Rui. Study on the Dynamic Prediction Method of ECD in Horizontal Well Drilling. Science Discovery. Vol. 7, No. 1, 2019, pp. 32-38. doi: 10.11648/j.sd.20190701.18

Received: March 17, 2019; Accepted: April 11, 2019; Published: April 28, 2019

\begin{abstract}
Well pressure and ECD control is one of the most important things in horizontal well drilling. This paper establish horizontal borehole pressure and ECD management considered seaside, temperature, drill pipe eccentric, drill pipe rotation and cutting bed though research horizontal well drilling. Sensitive factors like drill fluid density, drill fluid viscosity and so on has been found though analyzed the sensitive of ECD to drilling parameter. ECD real-time correction with different condition has been proposed though dimensionless comparison of factors. The first correction based on measured export parameters on the ground and use pump pressure to verify the correction. The second correction use trial method after compared pressure curve measured in down hole with model curve, verify the correction by pattern contrast. Precise ECD data can obtained use real-time correction mentioned to correct ECD management, ensure drilling safety in narrow density window.
\end{abstract}

Keywords: Horizontal Well, Well Pressure, ECD, Real-Time Correction

\section{水平井钻井过程中 ECD的动态预测方法研究}

\author{
马贤明 ${ }^{1}$, 管志川 ${ }^{1 *}$, 罗鸣 ${ }^{2}$, 李文拓 $^{2}$ ，潘瑞 ${ }^{1}$ \\ 1中国石油大学石油工程学院, 青岛, 中国 \\ ${ }^{2}$ 中海石油(中国)有限公司湛江分公司, 湛江, 中国
}

\section{邮箱}

568238622@qq.com (马贤明), guanzhch@upc.edu.cn（管志川）

\begin{abstract}
摘要: 水平井钻井过程中, 尤其是钻遇窄压力窗口复杂地层, 井筒压力及 $\mathrm{ECD}$ 的精确预测及控制是关乎安全高效钻井的 关键之一。本文针对水平井钻井特点, 建立了综合考虑井筒传热, 钻柱偏心, 钻柱旋转以及岩屑床等因素的水平井井筒 压力及ECD计算模型, 并通过计算不同条件下ECD的变化规律, 分析了ECD对相关钻井参数的敏感性, 确定了ECD的主 要敏感因素: 钻井液密度, 钻井液粘度, 钻井液排量等。在此基础上, 采用无量纲方法、依据现场实际情况, 提出了ECD 的实时修正方法: 以地面百压作为校正指标，通过预测不同计算模型参数条件下近钻头处的 $\mathrm{ECD}$ 并将之与实测值进行对 比和校正，依据不同的工况，依次对相关参数进行修正，得到较为精确的结果， ECD误差不超过 0.005 。采用本文建立的 模型对井筒压力进行预测, 具有较高的预测精度, 对提高水平井钻井井筒压力的预测精度和控制有重要意义。
\end{abstract}

关键词: 水平井, 井筒压力, $\mathrm{ECD}$, 实时修正 


\section{1. 引言}

在钻水平井过程中, 窄安全压力窗口时刻制约和威胁 着钻井工作的进行。对于窄压力窗口问题, 最主要的解决 方法是通过精确预测和控制ECD。ECD受温度, 钻柱旋转, 钻柱偏心等多方面因素的影响。1996年, 汪海阁等人建立 了水平井水平段环空压耗的计算公式, 主要考虑了水平段 岩屑传输的问题。2010年E.M.Ozhayoglu等人提出了非牛 顿流体在钻柱旋转下环空压耗的修正因子。2016年, oney Erge等人研究了水平井和大位移井钻柱偏心对 ECD的影 响。本文通过综合考虑钻柱偏心, 旋转以及岩屑床的影响。 建立ECD的动态预测方法以及实时修正方法。解释了窄安 全窗口下水平井 $E C D$ 变化规律, 为窄安全窗口下水平井钻 井提供了实用的参考, 最终实现在窄安全窗口下安全钻进 的目的。

\section{ECD计算模型的建立}

ECD(Equivalent Circulation Density)即当量循环密度。 当量循环密度如公式(1)[3] 是当量静态密度(ESD)和附加 当量循环密度之和(AECD公式(2))。

$$
\begin{gathered}
E C D=E S D+A E C D \\
A E C D=\frac{P_{\mathrm{a}}}{g H}
\end{gathered}
$$

$\mathrm{P}_{\mathrm{a}}$ 是到从井口到计算深度位置总的环空压耗, $\mathrm{Pa}$ 。对 ECD的精确预测的关键在于对环空压耗的计算。

(1)温度对 ECD的影响表现在温度升高使得钻井液体 积膨胀, 减小钻井液密度从而减小ECD。压力作用则相反, 压缩钻井液体积, 增加ECD。温度和压力对密度的影响经 验公式(3)如下

$$
\rho_{f}{ }^{\prime}=\rho_{f} e^{-4.3815 \times 10^{-6}(P-0.1)-2.3489 \times 10^{-4}(T-15)}
$$

$\rho_{f}$ 是初始钻井液密度, $\mathrm{g} / \mathrm{cm}^{3} ; \rho_{f}$ '是当前温度压力 下的钻井液密度, $\mathrm{g} / \mathrm{cm}^{3} ; \mathrm{P}$ 是计算点处的压力, $\mathrm{MPa}, \mathrm{T}$ 是计算点处的温度, ${ }^{\circ} \mathrm{C}$ 。

(2)为简化计算环空压耗使用了汪海阁提出的经验模 型公式(4)[1]

$$
\begin{aligned}
& \Delta P_{\text {bed }}=\frac{0.0260686 \mathrm{H}_{\mathrm{Tcb}} \Delta P_{\text {nobed }}}{f}\left[\frac{V_{a}}{g\left(d_{0}-d_{i}\right)(S-1)}\right]^{-1.25} \\
& +\left(1+0.00581695 H_{T c b}\right) \Delta P_{\text {nobed }}
\end{aligned}
$$

其中 $\Delta P_{\text {bed }}$ 是存在岩屑床情况下单位长度的环空压 耗, $\mathrm{Pa} ; \mathrm{H}_{\mathrm{Tcb}}$ 是无因次岩屑床高度; $\Delta P_{\text {nobed }}$ 是无岩屑床情 况下的单位长度环空压耗, $\mathrm{Pa} ; \mathrm{d}_{0}, \mathrm{~d}_{\mathrm{i}}$ 分别是环空的外径, 内径, $\mathrm{m} ; \mathrm{S}$ 是岩屑密度与钻井液密度之比; $\mathrm{v}_{\mathrm{a}}$ 是环空流体
流速, $\mathrm{m} / \mathrm{s} ; \mathrm{f}$ 是达西韦斯巴赫系数, $f=\frac{64}{\mathrm{Re}}$ (层流) $f=\frac{0.316}{\operatorname{Re}^{0.25}}$ (紊流)。

郑强, 王春艳[12]等人研究指出, 当环空流速小于临 界流速时在井斜角大于 30 度的井段之中会产生岩屑床。

(3)岩屑床对ECD的影响主要是减小环空流体的过流 断面, 从而增加ECD。岩屑床高度计算公式(5)(6)(7)如下 [1]

$$
\begin{gathered}
T_{\mathrm{c}}=0.015 d_{0}\left(\mu_{e}+6.15 \mu_{e}^{0.5}\right)(1+0.587 \varepsilon)\left(V_{c}-V_{a}\right) \\
\mu_{e}=K\left(\frac{2 n+1}{3 n}\right)^{n}\left(d_{0}-d_{i}\right)^{1-n}\left(12 V_{a}\right)^{n-1} \\
V_{c}=0.55\left(\frac{\rho_{s}-\rho_{f}}{\rho_{f}} D_{s}\right)^{0.667}\left(\frac{1+0.71 \theta+0.55 \sin 2 \theta}{\left(\rho_{f} \mu_{e}\right)^{0.333}}\right)
\end{gathered}
$$

$\mu_{e}$ 是钻井液有效粘度, $\mathrm{Pa}-\mathrm{s} ; \mathrm{v}_{\mathrm{c}}$ 是临界流速, $\mathrm{m} / \mathrm{s} ; \mathrm{K}$ 是钻井液稠度系数; $\rho_{s}$ 是岩屑密度, $\mathrm{g} / \mathrm{cm}^{3}$ 。通过上式计 算出岩屑床高度后, 与环空外径的比值作为无因次岩屑床 高度代入压耗公式计算。

(4)除岩屑床外还需要考虑钻柱偏心和旋转的影响。一 般来说, 钻柱偏心会减小ECD, 这点在层流中比紊流中更 明显。目前常用的估算钻柱偏心对ECD的影响的计算方法 主要是Narrow Slot理论。Suzana等人[4]经过实验分析和油 田数据验证得出结论, 完全偏心的环空压耗相比Narrow Slot理论计算的环空压耗要显著地减小, 有时候甚至超过 $50 \%$ 。因此他们提出了一种新的修正方法如公式(8), (9) 层流

$$
k_{2}=1-0.072 k^{0.8454} \frac{\varepsilon}{n}-1.5 k^{0.1852} \varepsilon \sqrt{n}+0.96 k^{0.2527} \mathcal{E} \sqrt[3]{n}
$$

紊流

$$
k_{2}=1-0.048 k^{0.8454} \frac{\varepsilon}{n}-0.666 k^{0.1852} \varepsilon \sqrt{n}+0.285 k^{0.2527} \varepsilon \sqrt[3]{n}
$$

$\mathrm{k}$ 是环空内径与环空外径之比; $n$ 是钻井液幂律指数; $\varepsilon$ 是偏心度。

(5)通过调研得知, 钻柱旋转对 ECD根据不同倾斜角的 井段影响不同。在井斜角小于 $70^{\circ}$ 时, 偏心钻柱旋转的轨 道运动会增加ECD, 而在井斜角大于 $70^{\circ}$, 特别是在有岩 屑床产生时, 钻柱的旋转运动会使更多的岩屑进入到悬浮 区[10]。从而清洁井眼, 因此会降低ECD的值。由于钻柱 旋转对 $\mathrm{ECD}$ 影响相关公式较为复杂实际情况难以计算, 因 此根据相关参数与 $\mathrm{ECD}$ 的关系作出如公式(10)假设

$$
k_{1}=\sqrt{\frac{1000 D_{s}}{N \varepsilon}}\left(0.7 \leq \mathrm{k}_{1} \leq 1.3\right)
$$

$D_{\mathrm{s}}$ 是平均岩屑粒径, $\mathrm{m} ; \mathrm{N}$ 是钻杆转速, $\mathrm{r} / \mathrm{min}$ 。 
(6)将影响因子带入无岩屑床存在时的单位长度环空 压耗为公式(11)

$$
\begin{aligned}
& \Delta P_{\text {nobed }}=k_{1} k_{2} k_{3} \frac{32\left(f_{f}+f_{s}\right) \rho_{f} Q^{2}}{\pi^{2}\left(d_{0}-d_{i}\right)^{3}\left(d_{0}+d_{i}\right)^{2}} \\
& +\rho_{s} g C u \cos \theta
\end{aligned}
$$

$\mathrm{K}_{3}$ 是根据实测数据的修正系数; $\mathrm{K}_{2}$ 是由于钻柱偏心对 $\mathrm{ECD}$ 造成影响的修正系数; $\mathrm{K}_{1}$ 是由于钻柱旋转对 $\mathrm{ECD}$ 造成 影响的修正系数; $f_{f}, f_{\mathrm{s}}$ 分别是流体摩擦系数和岩屑摩 擦系数; $\rho_{f}, \rho_{\mathrm{s}}$ 分别是钻井液密度和岩屑密度, $\mathrm{g} / \mathrm{cm}^{3}$; $C u$ 是环空固相浓度; $\mathrm{Q}$ 是钻井液排量, $\mathrm{m}^{3} / \mathrm{s} ; \theta$ 是井斜 角, 。

通过将各个影响因子带入公式(4)和公式(11)可以得 到环空压耗再结合公式(1)和公式(2)得到了修正后的ECD 计算模型。

ECD计算流程如下:

(1)划分计算段确定每段的钻进参数, 井身结构以及钻 柱参数等数据。

(2)根据计算段所处井深井斜角计算, 临界流速根据临 界流速与钻井液流速比较判断是否产生岩屑床。垂直段直 接用公式(11)计算，将井斜角设为 0 带入即可。

(3)当临界流速小于钻井液流速时, 认为计算段没有产 生岩屑床, 分别计算钻柱偏心, 旋转影响因子, 带入式(1-11) 计算得到计算段环空压耗。

(4)当临界流速大于钻井液流速时, 认为钻井液流速不 够将岩屑全部悬浮上返, 会在井壁产生岩屑床。计算存在 岩屑床的井段的环空压耗首先根据式(11)计算不存在岩屑 床时的换空压耗, 再根据式(5)计算无因次岩屑床高低, 最 后带入式(4)计算有岩屑床时的环空压耗。

(5)通过式(3)计算受温度压力影响的钻井液密度结合 计算得到的环空压耗根据式(1)式(2)得到计算段深度处的 ECD。

(6)重复过程2-5, 得到全井段的ECD。

\section{3. 参数敏感性分析}

\subsection{ECD对钻井参数敏感性研究}

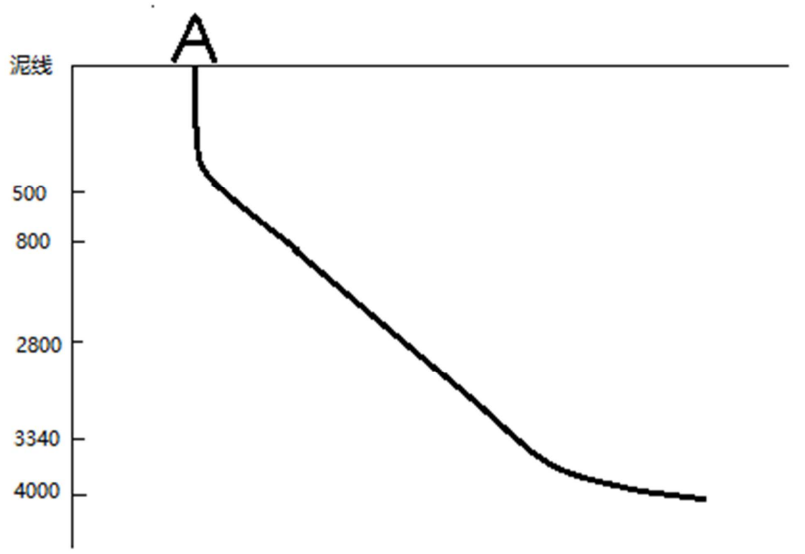

图1 井眼轨迹示意图。
实际钻井过程中井下情况时刻在变化, 因此通过对 $\mathrm{ECD}$ 进行实时的修正, 保证预测值的精确度。根据上述公 式对南海一口井井身结构进行计算, 井眼轨迹示意图如图 一所示钻具组合如表一所示, 通过在合理的变化范围内改 变相关钻井参数, 观察 $\mathrm{ECD}$ 变化规律, 进行相关参数的敏 感性分析。

(1)ECD随岩屑平均粒径变化图如图2所示

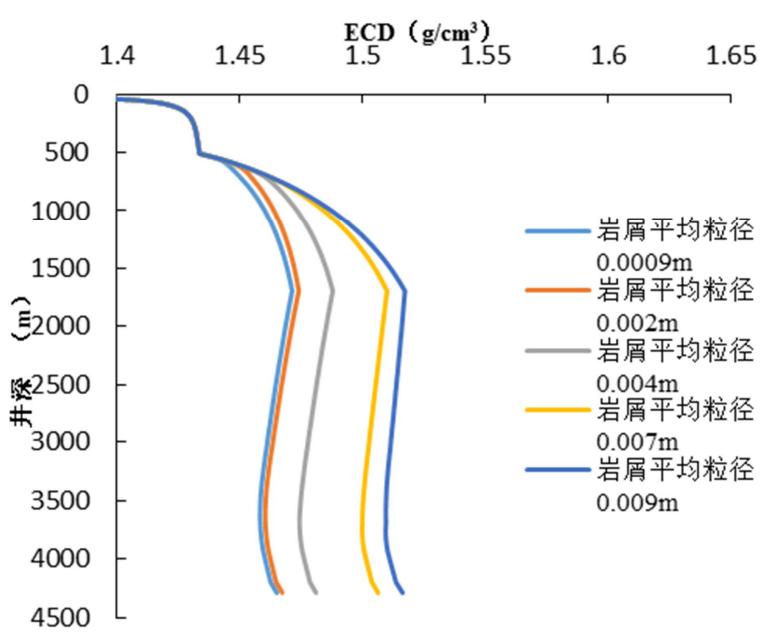

图2 全井 $\mathrm{ECD}$ 随岩屑平均粒径变化图。

从上图可以看出岩屑平均粒径大小主要影响倾斜段 和水平段的压耗, 随着岩屑粒径的减小, 环空摩阻下降, 岩屑更容易被钻井液带走, 有利于井眼清洁。

(2)ECD随钻井液排量变化图如图3所示

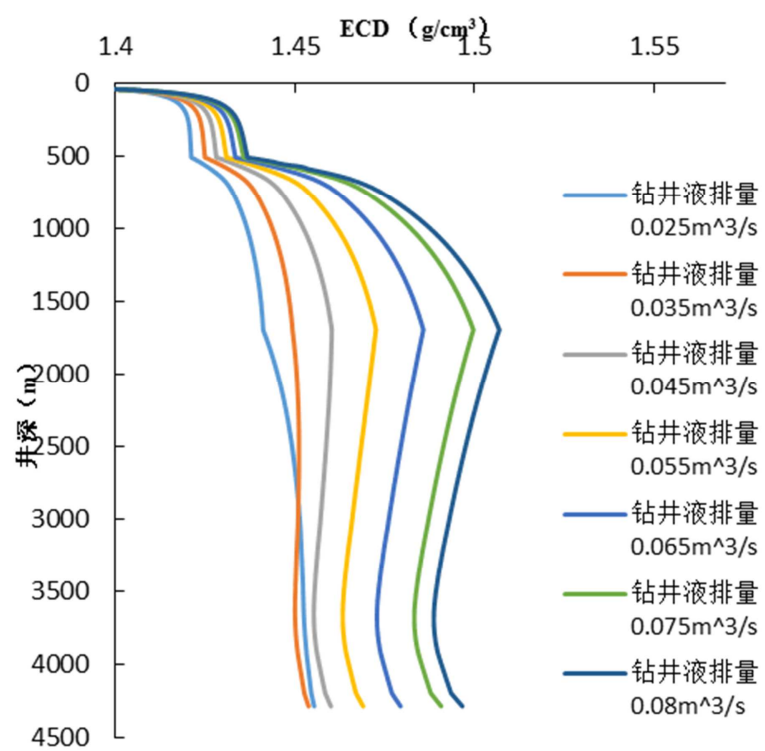

图3 全井ECD随钻井液排量变化图。

随着钻井液排量增加, 垂直段和一部分井斜角相对较 小的倾斜段的压耗上升, 而井斜角较大的倾斜段和水平段 压耗增加幅度较小。通常来说由于排量的增加会使整体的 $\mathrm{ECD}$ 上升, 但是水平段和倾斜角较大的井段存在岩屑床, 增加排量对岩屑床的清除起到了积极的作用。

(3)ECD随钻井液密度变化图如图4所示 


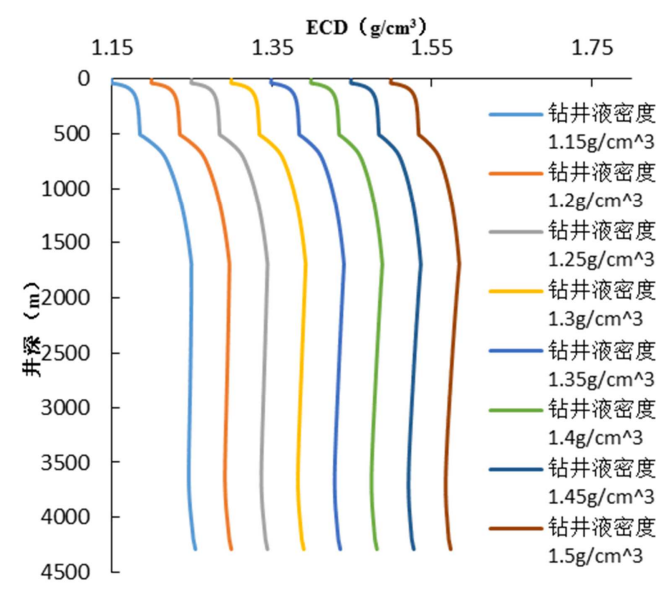

图4 全井ECD随钻井液密度变化图。

增加钻井液密度, 全井的ECD显著增加, 这是由于 $\mathrm{ESD}$ 的影响所以整井的ECD大幅增加, 近乎成线性增加。

(4)ECD随钻井液粘度图如图5 所示

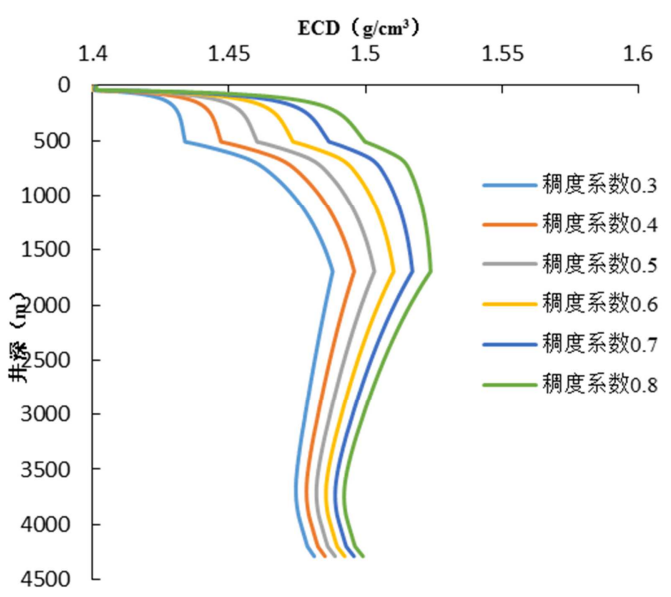

图5 全井 $\mathrm{ECD}$ 随钻井液粘度变化图。

ECD随着钻井液粘度增加而增加, 下部井斜角较大的 倾斜段和水平段由于钻井液粘度增加, 提高了携岩能力, 对井眼清洁起到了积极作用, 因此增加趋势较小。

(5)ECD随机械钻速变化图如图6所示

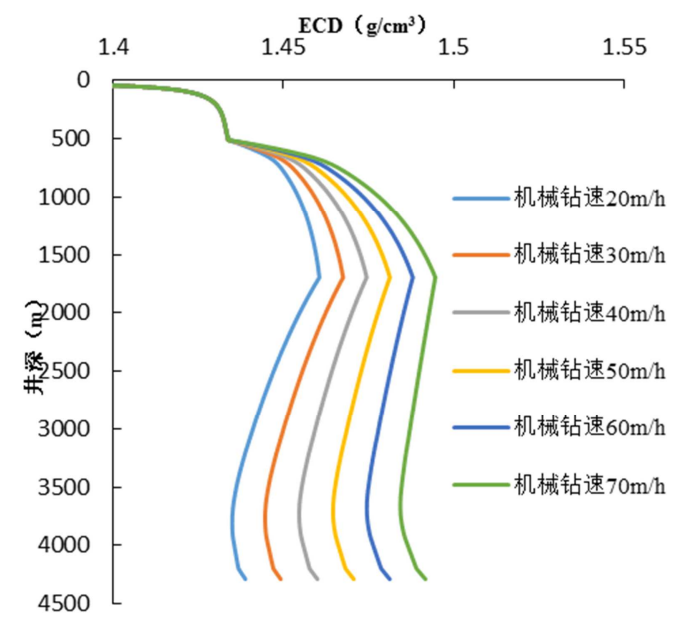

图6 全井段ECD随机械钻速变化图。
增加机械钻速, 主要增加了倾斜段和水平段的ECD, 特别是下部大斜度井段和水平段, $\mathrm{ECD}$ 增加幅度大, 这是 由于机械钻速的增加, 导致了环空岩屑浓度的增加, 而钻 井液排量没有变化, 因此井眼清洁度下降, 岩屑床高度增 加, 环空过流断面减小, 环空压耗大幅增加。

(6)ECD随钻杆转速变化图如图7所示

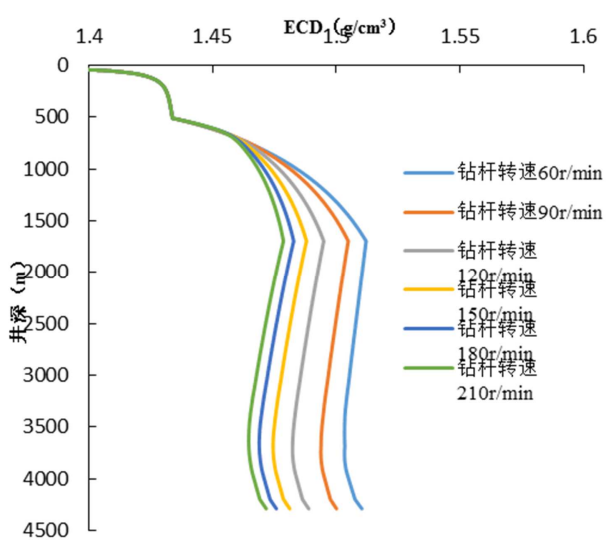

图7 全井段 $\mathrm{ECD}$ 随钻杆转速变化图。

可以看到钻杆转速对垂直段基本没有影响, 但是对倾 斜段影响十分大。增加钻杆转速, 倾斜段 ECD普遍下降, 这是由于钻杆转速增加, 将沉积在岩屑床部分的岩屑带到 悬浮区域。更有利于钻井液将岩屑带离井底, 井眼清洁度 提高, 环空压耗相应减小。

\section{2. 各参数的敏感性分析}

对钻井参数无因次化如公式(12)

$$
\text { 钻井参数 } L_{\mathrm{i}} \text { 无因次量 }=\frac{L_{\mathrm{i}}-L_{\min }}{L_{\max }-L_{\min }}
$$

对ECD做以下处理，如公式(13)

$$
E C D \text { 差值 }=\left|E C D-E C D_{L_{\min }}\right|
$$

以无因次数为 $\mathrm{X}$ 轴, $\mathrm{ECD}$ 差值为 $\mathrm{Y}$ 轴做曲线, $\mathrm{Y}$ 值越大 说明对应参数在这一井段对 ECD影响越大。分别对垂直 段，两段倾斜段，水平段进行分析。

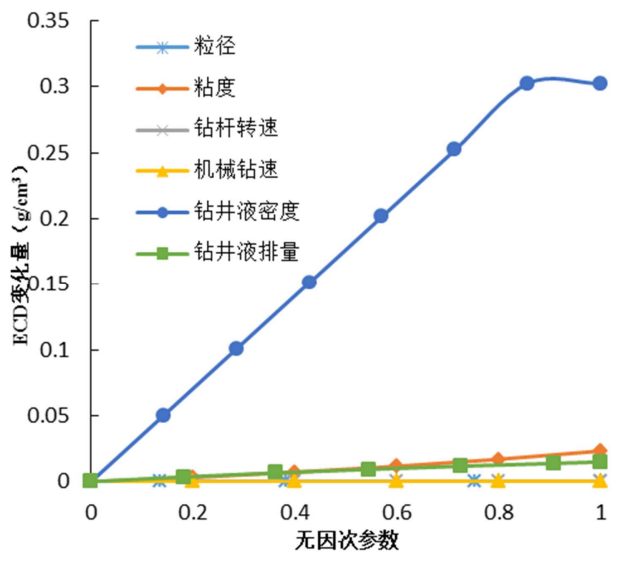

图8 垂直段钻井参数无因次曲线。 


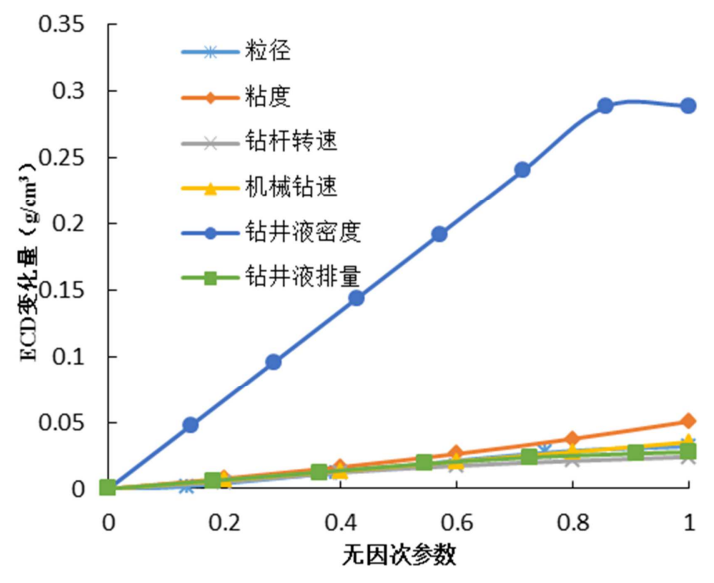

图9 倾斜段钻井参数无因次曲线。

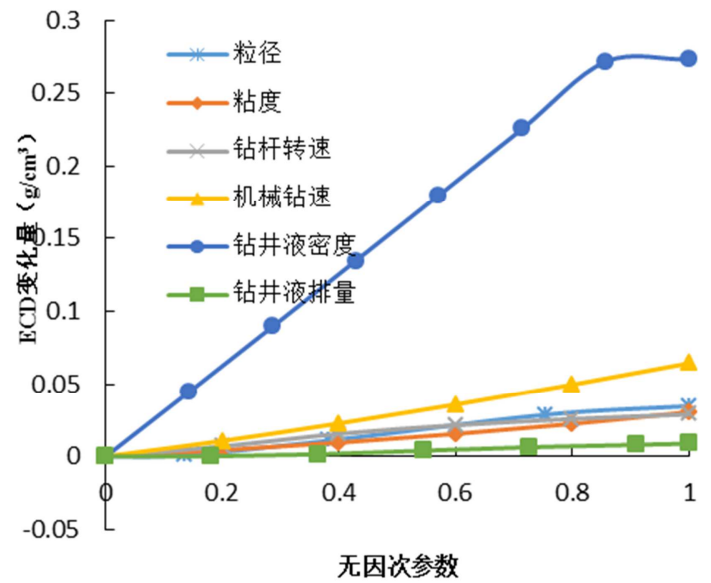

图10 倾斜段处钻井参数无因次曲线。

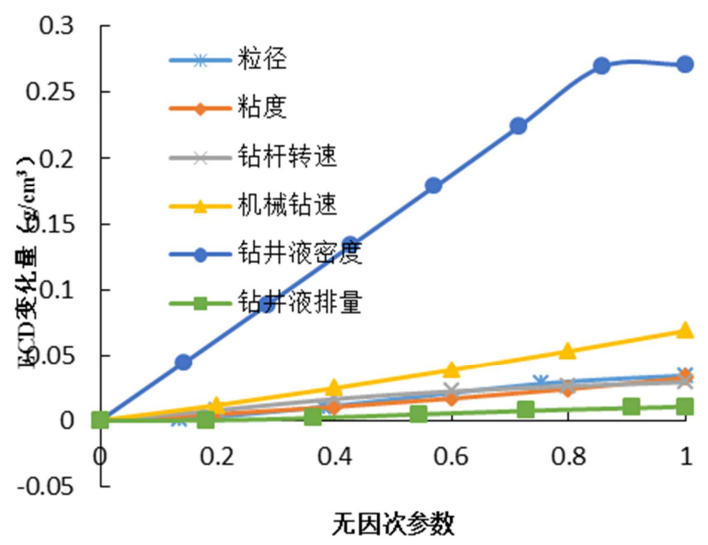

图11 水平段钻井参数无因次曲线。

通过分别对垂直段，两段倾斜段以及水平段进行分析 可以发现, 对ECD影响最大的是钻井液密度, 这也映证了 合适的钻井液密度是保证安全钻进的重要手段之一, 垂直 段ECD对钻井液排量和粘度也较为敏感。倾斜段根据流型 不同, 分为两种。层流时, ECD对粘度影响较为敏感, 其 次是粒径和机械钻速最后是钻杆转速和排量。在紊流时, 机械钻速较为敏感, 其次是粒径和钻杆转速以及粘度, 最 后是钻井液排量。水平段与倾斜段紊流状态相似。

\section{ECD实时修正方法}

利用以上水平井ECD计算模型计算南海一口海上井 的ECD数值, 在相同的钻井参数和钻具组合情况下如表 1 , 表2, 利用计算不同深度处ECD值与PWD实测值对比如下 图11所示。

表1 实测钻具组合。

8-1/2’PDC·Bit+旋转导向工具(带浮

阀)+6-3/4"LWD+6-3/4"MWD+6-3/4"NMDC+堵漏短节

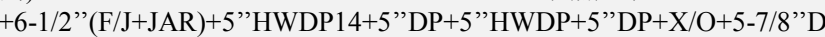
$\mathrm{P}$

表2 实测钻井参数。

\begin{tabular}{|c|c|c|c|c|}
\hline $\begin{array}{l}\text { 钻 井 液 性 } \\
\text { 能·g/ } / \mathrm{cm}^{3} / \mathbf{m P a . s} / \mathbf{P a}\end{array}$ & $1.30 / 40 / 10$ & $1.30 / 50 / 15$ & $1.30 / 40 / 10$ & $1.30 / 50 / 15$ \\
\hline $\begin{array}{l}\text { 排量/机械钻速/转速 } \\
\text { /水眼 }\end{array}$ & \multicolumn{2}{|c|}{$\begin{array}{l}4000.00 \cdot \mathrm{L} / \mathrm{min} / 60 \mathrm{~m} / \mathrm{h} / 1 \\
50 \mathrm{RPM} / 9 * 14\left(1.353 \mathrm{in}^{2}\right)\end{array}$} & \multicolumn{2}{|c|}{$\begin{array}{l}2000.00 \cdot \mathrm{L} / \mathrm{min} / 40 \mathrm{~m} / \mathrm{h} / 1 \\
20 R P M / 7 * 14\left(1.052 \mathrm{in}^{2}\right)\end{array}$} \\
\hline 泵压·psi & 3422.01 & 3751.63 & 2810.45 & 3196.39 \\
\hline 钻头压耗·psi & 609.33 & 609.33 & 251.82 & 251.82 \\
\hline 钻具压耗·psi & 2519.58 & 2759.25 & 1773.15 & 1951.06 \\
\hline 环空压耗·psi & 193.10 & 283.05 & 685.48 & 893.51 \\
\hline $\mathrm{ECD} \mathrm{g} / \mathrm{cm}^{3}$ & 1.38 & 1.41 & 1.513 & 1.57 \\
\hline
\end{tabular}

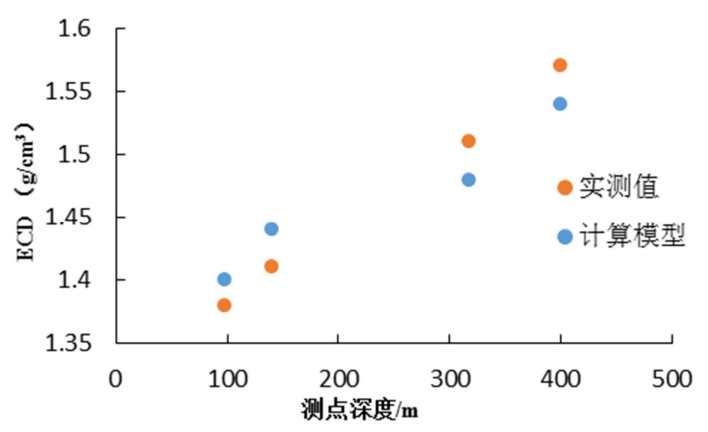

图12 现场实测值与模型值对比图。

从对比图可以看出, ECD预测精度不够准确, 在排量 水眼较大的情况下预测值偏高, 排量, 水眼较小情况下预 测值偏低。因此需要对ECD预测模型进行实时修正。

根据前文对钻井参数敏感性研究确定了在不同井段 中各参数影响因素的大小, 根据实测 $\mathrm{ECD}$ 与计算值进行比 较, 根据其差值选取修正参数, 进行有方向性的试算。对 上例进行修正由于计算值与实际值差值较大，根据敏感性 分析可知, 首先对机械钻速进行试算法修正如图 12, 图13。 以实测 $\mathrm{ECD}$ 为 1.41 为例。

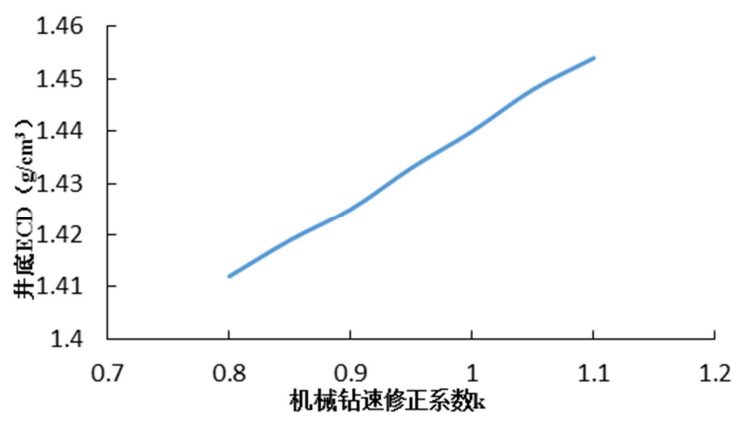

图13 机械钻速修正系数 $\mathrm{k}$ 与井底 $\mathrm{ECD}$ 关系图。 


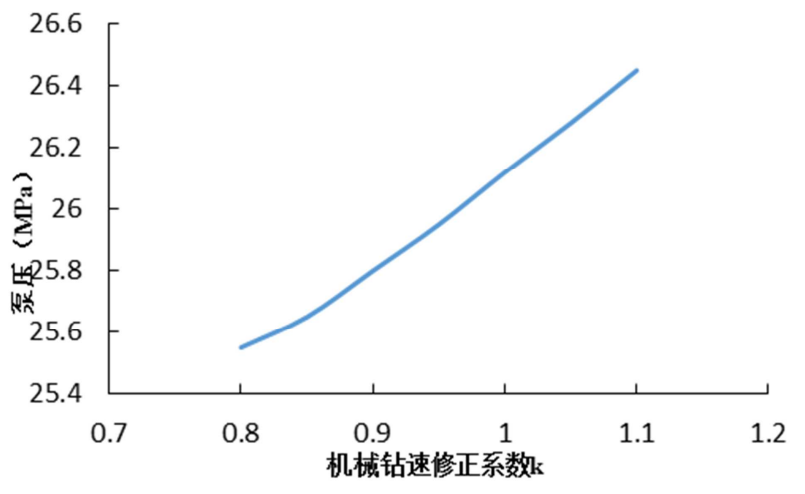

图14 机械钻速修正系数 $\mathrm{k}$ 与脣压关系图。

由上图可以看出, 当修正机械钻速使井底 $\mathrm{ECD}$ 接近实 测值达到 1.41 左右时, 泵压为 $25.5 \mathrm{MPa}$, 略低于实际情况, 由敏感性分析可知当钻井液粘度增加时, 葲压上升, 其对 $\mathrm{ECD}$ 影响较小。因此对钻井液粘度进行修正如下图14, 图 15 。

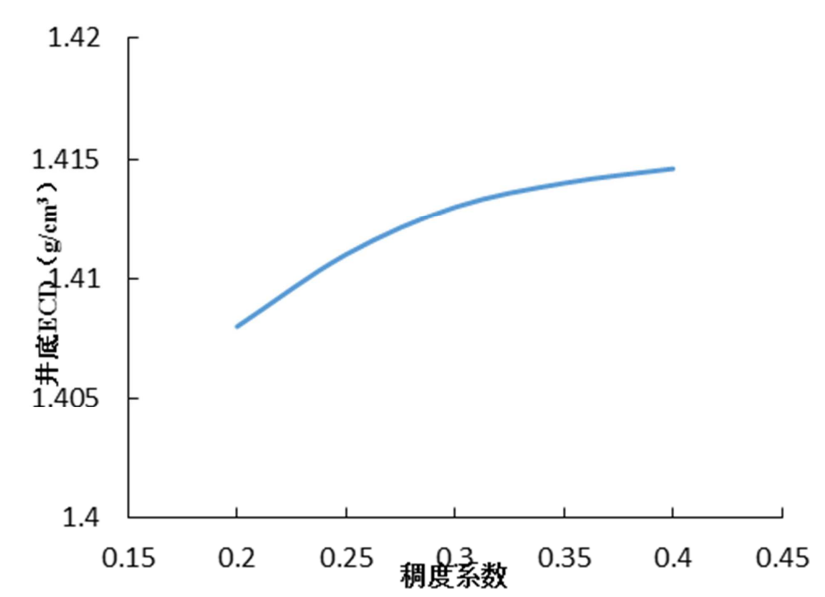

图15 稠度系数与井底 $\mathrm{ECD}$ 关系图。

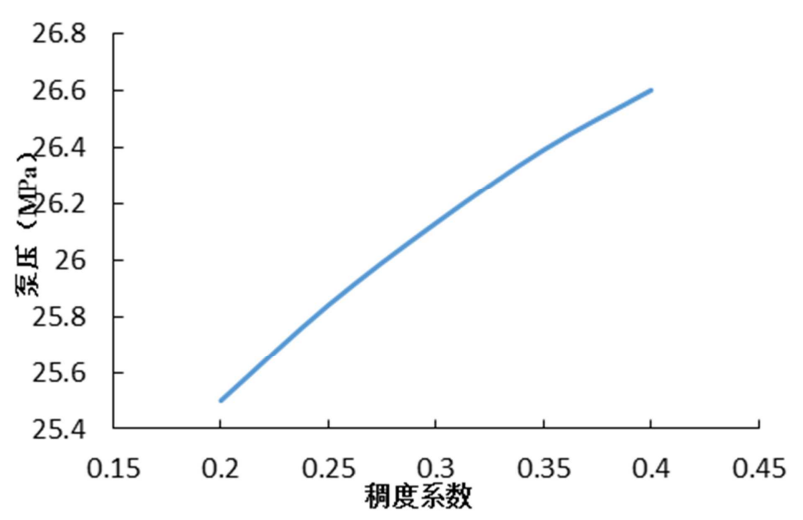

图16 稠度系数与泵压关系图。

由上图可知对粘度进行修正后, 原压和 $\mathrm{ECD}$ 与实际值 已经较为接近。

最终通过修正得到 $\mathrm{ECD}$ 与实际的差值不超过 0.004 , 泵压与实际差值不超过 $0.1 \mathrm{MPa}$ 。得到了较为精确的结果。 运用相同的方法分别对其他三种工况下的ECD进行 修正得到修正后的对比图如下图16所示。

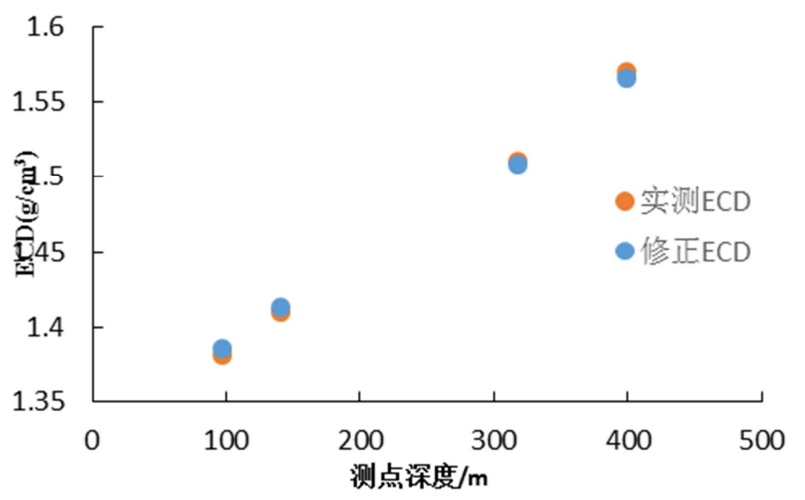

图17 现场实测值与模型值修正后对比图。

通过上图可以看出修正后的ECD模型计算值与实测 值基本相符, ECD误差不超过 0.005 , 原压误差不超过 $0.2 \mathrm{MPa}$ 。总结修正方法如下:

(1) 当ECD与实测值差值大于 0.3 考虑对钻井液密度进行 修正。

(2) 当 $\mathrm{ECD}$ 与实测值差值小于 0.3 或者通过对钻井液修正 后差值小于 0.3 , 继续对机械钻速进行修正直至差值 小于 0.1 , 随后观察洜压与计算值差值。

(3) 通过洜压与计算值差值进行有方向性的修正钻井液 粘度, 直至ECD误差小于 0.05 , 泵压误差小于 $1 \mathrm{MPa}$ 。 通过以上的修正方法, 使模型计算值达到较高的精 度。在钻井过程中, 通过记录不同深度的ECD和洜压值与 计算值进行对比修正得到全井段的ECD值, 不仅保证了近 钻头处的ECD控制与监测, 同时也保证了上部井段的安全 性。

\section{5. 结论}

本文利用经验公式建立了考虑, 温度, 钻柱偏心, 钻 柱旋转以及岩屑床的水平井钻井过程中 ECD动态预测方 法。分析了ECD对各个参数的敏感性发现钻井液密度是影 响 $\mathrm{ECD}$ 最重要的因素, 其次是钻井液排量和粘度, 通过对 水平井井段进行划分解释了不同井段各钻井参数对 ECD 的影响规律和影响大小。通过对南海一口井进行计算, 通 过与实际计算值进行对比提出了对模型计算值的修正方 法。以泵压作为修正指标验证修正的正确性。通过修正达 到了较为精确地结果。通过本文的研究, 为水平井钻井过 程中井筒压力和 $\mathrm{ECD}$ 预测模型的实时修正提供了理论基 础, 提高了模型的预测精度, 有利于窄密度窗口条件下水 平钻井井筒压力和 $\mathrm{ECD}$ 的控制, 降低钻井风险。

\section{参考文献}

[1] 汪海阁, 刘希圣等.水平井水平井段环空压耗模式的建立. 石油大学学报(自然科学版), 1996, 2(2): 30-35.

[2] 汪志明, 郭晓乐等.南海流花超大位移井井眼净化技术.石油 钻采工艺，2006，28(1): 4-8. 
[3] 王鄂川, 樊洪海等.环空附加当量循环密度的计算方法.断块 油气田, 2014,24(5): 671-674.

[4] Suzaba santos costa,Sidney stuckenbruck.Simulation of Transient Cuttings Transportation and ECD in Wellbore Drilling. SPE 113893.

[5] Ramadan Ahmed and Stefan Miska. Experimental Study and Modeling of Yield Power-Law Fluid Flow in Annuli with Drillpipe Rotation. IADC/SPE 112604.

[6] E.M.Ozbayoglu and M.Sorgun.Frictional Pressure Loss Estimation of Non-Newtonian Fluids in Realistic Annulus with Pipe Rotation. JCPT, 2010, 49(12):57-64.

[7] Terry Hemphlii and Krishna Ravi, Halliburton. Imporved Prediction of ECD with Drill Pipe Rotation. IPTC 15424.

[8] T N Ofei, S Irawan and W Pao. Cuttings-liquid frictional pressure loss model for horizontal narrow annular flow with rotation drillpipe. IOP Conf. Series: Material science and Engineering 78(2015) 012009.

[9] Oney Erge,Ali Karimi Vajargah,Mehmet Erren Ozbayoglu, Eric Van Oort. Improved ECD Prediction and Management in Horizontal and Extended Reach Wells with Eccentric Drillstrings.
[10] 孙晓峰, 纪国栋等.大斜度井偏心环空对岩屑运移的影响. 特种油气藏, 2015, 22(6): 133-136.

[11] 封卫强, 李琪等. 钻井液循环密度当量的分析. 价值工程, 2012, 0402202: 22-23.

[12] 郑强, 王春艳.岩屑床的形成机理及对环空ECD的影响.价值 工程, 2012, 24002902: 29-30.

[13] 宋洵成, 管志川等.斜井岩屑运移临界环空流速力学模型. 中国石油大学学报(自然科学版), 2009,33(1): 53-56.

[14] 管志川.温度和压力对深水钻井油基钻井液液柱压力的影 响.石油大学学报(自然科学版), 2003, 27(4): 48-52.

[15] 汪志明, 张政.水平井两层稳定岩屑传输规律研究. 石油大学 学报(自然科学版), 2004, 28(4): 63-66.

[16] 杨雪山, 李胜等.水平井井筒温度场模型及ECD的计算与分 析.钻井液与完井液2014，31(5): 63-66.

[17] 张好林, 李根生等.水平井井筒清洁临界流速简化模型.钻采 工艺, 2014,37(4): 5-8. 